\title{
Métodos analítico e participativo para avaliação de usabilidade: estudo comparativo
}

\author{
Analytical and participatory methods for usability evaluation: a comparative \\ search
}

REIS, Priscila Câmara; Bacharel; Universidade Federal do Rio Grande do Norte

Priscila.camarareis@gmail.com

BASTOS, Helena Rugai; Doutora; Universidade Federal do Rio Grande do Norte

helenarugai@gmail.com

\section{Resumo}

As avaliações de usabilidade são imprescindíveis no contexto da Educação a Distância [EAD], pois permitem o constante exame dos níveis de interação e garantem o funcionamento eficaz dos sistemas utilizados. Diante disso, este artigo estabelece uma comparação entre dois métodos de análise de usabilidade, a avaliação analítica e a avaliação com abordagem participativa. Ambos foram aplicados na avaliação do cadastro e primeiro acesso ao portal SENAI EAD, especificamente para realizar matrícula em curso livre de Empreendedorismo, oferecido pela instituição. A avaliação analítica foi realizada pelos avaliadores, autores da pesquisa, enquanto a participativa contou com um grupo de usuários, estabelecidos a partir da análise do perfil de interessados publicado no portal da instituição. A aplicação das duas abordagens possibilitou observar as diferenças entre as duas avaliações, quais foram os problemas encontrados em cada uma das fases do processo, a natureza destes problemas e, ainda, a eficiência de cada abordagem, tendo em vista a usabilidade do sistema estudado.

Palavras Chave: usabilidade, avaliação analítica, avaliação participativa.

\begin{abstract}
Usability evalutions are essential in the context of $E A D$, since they guarantee the efficient operation of the systems used. This study presents a comparative analysis between two methods of usability analysis: an analytical evaluation and an evaluation with a participatory approach. Both were applied while evaluating both registration and first access to the SENAI EAD portal, especially to undertake free course enrollment, named Entrepreneurship. The analytical assessment was made by the evaluators themselves while the participatory one counted on a group of users, chosen from the stakeholder profile analysis published on the institution's website. The research made it possible to observe the differences between the two evaluations, which problems were found in each of them, including the nature of the problems and and even verify which one was more productive considering the system usability and the accessed pages.
\end{abstract}

Keywords: usability; analytical evaluation; participatory evalution 


\section{Introdução}

A Educação a Distância [EAD] vem crescendo no Brasil e no mundo de maneira significativa. Tanto o avanço tecnológico - como por exemplo a Internet mais rápida - quanto o fácil acesso à informação, têm contribuído para este crescimento (MORÁN, 2015). Nessa perspectiva, é possível observar o crescimento da EAD, sobretudo de alunos matriculados em cursos livres coorporativos ou não no país. De acordo com dados da Associação Brasileira de Educação a Distância (CENSO EAD.BR, 2016), o número de matrículas no período compreendido entre 2016 a 2017 foi de 709.853 alunos. Ainda segundo o Censo EAD.BR 2016 a maioria dos cursos livres coorporativos são elaborados para empresas, principalmente, para o treinamento operacional de funcionários e às demandas específicas de clientes. Já os cursos não vinculados a uma empresa são, na sua maioria para a iniciação ou atualização profissional. De fato, o ensino a distância permite o acesso à formação continuada e complementa a formação oferecida pela educação formal. Igualmente, contribui para minimizar questões sobre acesso ao ensino, que envolve o número de vagas oferecidas pelas redes de ensino, que são restritas, e a necessidade de incluir socialmente uma maior parcela de alunos (FILATRO, 2004).

Nesse cenário, é cada vez maior a necessidade de profissionalizar aqueles que se dedicam à criação de soluções para o aprendizado eletrônico, o que inclui a qualidade de materiais e recursos para o Ambiente Virtual de Aprendizagem [AVA], em razão da competitividade nesse campo de trabalho, mas principalmente pelo fato de os alunos e professores estarem, nestes ambientes, separados por espaço/tempo (FILATRO, 2008). Assim, é necessário pensar em soluções e no uso de recursos e de ferramentas de ensino que proporcionem uma aprendizagem clara e direta, o que pode ampliar horizontes e democratizar ainda mais, as barreiras do aprendizado. Nesse contexto, podemos considerar o Design Instrucional como peça chave para a criação de soluções eficientes para esta modalidade de ensino. Vale ressaltar que, de acordo com Filatro (2008), Design Instrucional ou Design de Aprendizagem pode ser definido como uma ação intencional e sistemática, que envolve planejamento, desenvolvimento e aplicação de técnicas, a fim de promover a aprendizagem humana.

Abordar o Design Instrucional [DI doravante] envolve diversas questões que articulam outras subáreas do design, como por exemplo, avaliar a usabilidade dos ambientes, das interfaces e dos materiais desenvolvidos. Os inúmeros tipos de avaliações podem incluir o estudo de diferentes métodos em diversas etapas do processo de concepção ou de aprimoramento dos materiais produzido para a EAD. De qualquer maneira, o objetivo maior é a criação e o desenvolvimento de materiais condizentes com a realidade dos alunos e que proporcionem uma aprendizagem significativa.

O artigo apresenta uma comparação entre dois métodos de análise de usabilidade - a avaliação analítica, realizada pelos autores do estudo, e a avaliação participativa, abordagem que envolveu no processo os usuários do sistema, o portal Senai EAD, em particular a etapa de cadastro e o primeiro acesso no curso de Empreendedorismo oferecido gratuitamente pela instituição neste ambiente. $O$ estudo aqui apresentado é resultante de pesquisa maior, que compreende o estudo e as articulações entre conceitos e fundamentos sobre Design Instrucional, Educação a Distância, Design de Interação, Design Centrado no Usuário, métodos e processos de avaliação de usabilidade. É importante mencionar que o portal SENAI EAD foi selecionado em razão do reconhecimento, do alcance e da variedade dos cursos a distância oferecidos pela instituição. Quanto à escolha do curso específico - Empreendedorismo, a seleção tem relação com o perfil dos usuários estabelecido no 
portal SENAI EAD e a expectativa do grupo de usuários que participaram da avaliação: estudantes de graduação de Design da região potiguar, com idade entre 19 e 21 anos, com interesse em fundamentos, conceitos, abordagens e ações relacionadas ao empreendedorismo e ligados, de alguma maneira, à indústria ou ao comércio do município de Natal - RN, onde vivem e trabalham.

Neste artigo descrevemos os procedimentos realizados nas duas avaliações e, igualmente, identificamos as qualidades dos métodos aplicados em cada fase dos processos, estabelecendo comparação entre eles.

\section{Abordagens, métodos e processos}

A pesquisa compreendeu o estudo sobre conceitos e fundamentos de Design Instrucional [DI], EAD, design de interação, Design Centrado no Usuário [DCU], métodos e processos analíticos de avaliação de usabilidade e abordagens participativas. Assim, para a estruturação das avaliações, os pesquisadores inicialmente abarcaram o contexto histórico da educação a distância no Brasil, sobretudo a partir de estudos realizados por Mugnol (2009) e Filatro (2004; 2008), Morán (2015) e dados oferecidos pela Associação Brasileira de Educação a Distância (ABED), em especial no Relatório Analítico da Educação a Distância no Brasil (CENSO EAD.BR, 2016). Em seguida, levou-se em conta as competências relacionadas ao campo do DI, articuladas aos conceitos do design de interação e usabilidade, sob a visão, principalmente, de Nielsen (1993), das autoras Preece, Rogers e Sharp (2013) e do International Board of Standards for Training, Performance and Instruction [IBSTPI]. O documento Instructional Design Competencies (2012), publicado pelo IBSTPI, por exemplo, considera 22 competências do Design Instrucional, que são agrupadas em cinco domínios: fundamentos da profissão, planejamento e análise, design e desenvolvimento, avaliação e implementação, gerenciamento. As 22 competências podem ser verificadas a partir de um total de 105 proposições de desempenho que devem ser analisados. Já no contexto do design de interação, Preece, Rogers e Sharp (2013) pressupõem quatro etapas principais articuladas para desenvolvimento de interfaces. São elas: estabelecer requisitos, criar alternativas de design, avaliar e prototipar. Para este estudo, foram observadas com maior ênfase as competências e os conceitos de design de interação relacionados à etapa ou o domínio da avaliação.

Tais processos de avaliação de interação levaram em conta objetivos que envolvem: as necessidades dos usuários e a compreensão dos designers sobre essas necessidades; a identificação de problemas no processo de interação, o que pressupõe a análise da interface, para identificar como sua configuração afeta o acesso às informações e o trabalho dos usuários; estudo de alternativas para a configuração da interface; levantamento e análise de métricas de usabilidade, que também considera aspectos ergonômicos (PREECE, ROGERS, SHARP, 2013).

Quanto à avaliação da interface do sistema existente disponibilizado pelo SENAI EAD, como mencionado, o estudo envolveu duas etapas e dois métodos distintos com enfoque na usabilidade, o que permitiu avaliar a qualidade do sistema estudado, considerando fatores prioritários como: facilidade e eficiência de uso; facilidade de aprendizado; aspectos funcionais e operacionais do sistema; satisfação do usuário; flexibilidade e segurança de uso (NIELSEN, 1993; PREECE, ROGERS, SHARP, 2013).

A primeira etapa do processo, a avaliação analítica, realizada pelos avaliadores, consistiu em examinar aspectos de usabilidade da interface do sistema - SENAI EAD - a partir de tarefas estabelecidas necessárias para a realização do cadastro e do primeiro acesso ao curso selecionado (PRATES e BARBOSA, 2013). Este tipo de avaliação requer o uso de método analítico denominado 
percurso cognitivo (WHARTON ET AL., 1994) e busca analisar a facilidade de aprendizado do sistema, com foco na exploração dos usuários. Nesse caminho, o percurso cognitivo contribui para a análise dos aspectos funcionais do sistema, no momento da realização das tarefas típicas disponibilizadas na interface. $\mathrm{Na}$ avaliação analítica por percurso cognitivo o custo do aprendizado deve ser determinado pelo benefício imediato aos seus usuários (PRATES; BARBOSA, 2003). Embora seja um método que não envolve os usuários, o percurso cognitivo visa prever hipóteses sobre o conhecimento dos usuários a respeito do sistema. Assim, os avaliadores devem criar uma lista de tarefas que os usuários devem percorrer, e a partir do momento em que avançam as etapas, verificar potenciais problemas de interface.

O processo analítico envolve a observação de funcionalidades do sistema a partir do reconhecimento e identificação de informações e recursos da interface, o que inclui a verificação de sua configuração, dos termos e do vocabulário utilizados. Igualmente, o método permite a investigação de feedbacks disponibilizados, resultantes das ações realizadas para acesso às informações e recursos da interface.

No estudo aqui apresentado foram identificados e classificados os problemas de usabilidade, para posterior comparação com as questões levantadas e classificadas na segunda etapa do processo de avaliação - a avaliação participativa.

A avaliação participativa foi baseada, para além dos autores e obras mencionadas (PREECE, ROGERS; SHARP, 2013; IBSTPI, 2012), em estudos sobre design participativo, usabilidade e Design Centrado no Usuário [DCU], a partir da consulta das obras de Santa Rosa e Moraes (2012), Lowdermilk (2013), Nielsen e Loranger (2007).

De acordo com Santa Rosa e Moraes (2012), a metodologia do design participativo, na qual os usuários atuam como Co projetistas, é adotada com o intuito de melhorar a qualidade do sistema, evitando funções sem utilidade e aumentando o nível de aceitação, para um uso mais efetivo dos sistemas. Nessa perspectiva, os usuários dão contribuições efetivas para o aprimoramento do sistema, que refletem suas perspectivas e necessidades de uso. Ao estabelecer comparação entre as duas abordagens - DCU e design participativo - Santa Rosa e Moraes (2012) destacam:

\begin{abstract}
Uma das principais diferenças entre o design participativo e a metodologia de designcentrado no usuário, é que o envolvimento dos usuários vai muito além da experimentação ou teste de alternativas de design, ou da participação de métodos qualitativos para medida da satisfação, facilidade de uso ou aceitação. No design participativo, os usuários podem participar do projeto e design como se fizessem parte da equipe de design. (SANTA ROSA; MORAES, 2012, p. 18).
\end{abstract}

Lowdermilk (2013), assinala que o DCU consiste em uma metodologia que, essencialmente, ajuda a criar sistemas de computação que atendam às necessidades dos usuários. Assim, o DCU assume papel de ferramenta, que garante ao usuário final uma boa usabilidade. Para Lowdermilk (2013) as avaliações de usabilidade são recursos-chave do DCU, que podem ajudar a fornecer respostas e determinar linhas de base para acompanhar melhorias no design de um sistema.

É possível observar, que para as duas abordagens, os autores destacam a necessidade de avaliações de uso ou usabilidade dos sistemas. Para tanto, recomendam a compreensão e a aplicação de critérios ergonômicos, que contribuam para a utilização do sistema de maneira produtiva, com conforto e segurança.

Levando em conta o objeto de estudo e a avaliação da etapa de cadastro e o primeiro acesso 
ao curso de Empreendedorismo do SENAI EAD, foi necessário estabelecer os critérios ergonômicos, que facilitassem ou garantissem o aprendizado no ambiente estudado. Tais critérios foram utilizados para a condução do processo de avaliação tanto analítica quanto participativa. Na Tabela 1 , pode-se verificar quais são esses critérios e como são definidos.

Tabela 1 - Critérios ergonômicos para a avaliação de usabilidade

\begin{tabular}{ll}
\hline Critérios Ergonômicos & Descrição \\
\hline Condução & $\begin{array}{l}\text { Meios disponíveis para orientar, informar e conduzir o usuário na } \\
\text { interação com o computador; }\end{array}$ \\
\hline Carga de Trabalho & $\begin{array}{l}\text { Relaciona todos os elementos da interface que têm papel importante } \\
\text { na redução da carga cognitiva e perceptiva do usuário e no aumento da } \\
\text { eficiência do diálogo; }\end{array}$ \\
\hline Controle explícito do usuário & $\begin{array}{l}\text { Refere-se tanto ao processamento explícito das ações do usuário pelo } \\
\text { sistema, quanto ao controle que este tem sobre o processamento de } \\
\text { suas ações pelo sistema; }\end{array}$ \\
\hline Adaptabilidade & $\begin{array}{l}\text { Capacidade do sistema de reagir conforme o contexto, as necessidades } \\
\text { e preferências do usuário; }\end{array}$ \\
\hline Gestão de erros & $\begin{array}{l}\text { Mecanismos que evitam ou reduzem a ocorrência de erros e, quando } \\
\text { eles ocorrem, favorecem sua correção; }\end{array}$ \\
\hline Homogeneidade/coerência & Forma como as escolhas, na concepção da interface é conservada; \\
\hline Significado de códigos e denominações & $\begin{array}{l}\text { Diz respeito à adequação entre o objeto ou a informação apresentada } \\
\text { ou pedida e sua referência; }\end{array}$ \\
\hline Compatibilidade & $\begin{array}{l}\text { Acordo entre as características do usuário, as tarefas e a organização } \\
\text { das saídas, das entradas e do diálogo de uma dada aplicação. Diz } \\
\text { respeito, ainda, ao grau de similaridade entre diferentes ambientes e } \\
\text { aplicações. }\end{array}$ \\
\hline
\end{tabular}

Fonte: adaptação do texto de Godoi e Padovani (2009).

\subsection{Avaliação Analítica}

Entende-se por avaliação analítica uma avaliação de usabilidade, a partir da qual os avaliadores examinam aspectos de uma interface, que será utilizada por um outro usuário (MACK; NIELSEN, 1994). Ela tem a finalidade de identificar problemas de usabilidade de uma interface e selecionar aqueles que devem ser corrigidos. Como mencionado, para esta etapa de avaliação foram estabelecidas, a partir de método que analisa o percurso cognitivo, tarefas específicas necessárias para acesso ao sistema e às informações da interface para realizar as ações pretendidas, o cadastro e primeiro acesso ao curso selecionado (WHARTON, et al., 1994). Levando em conta o portal SENAI $E A D$, para os usuários conseguirem realizar a matrícula no curso de Empreendedorismo, é necessário acessar páginas iniciais e gerais do sistema.

Considerando o objetivo estabelecido - realizar a matrícula no sistema e curso selecionados -, a partir da observação dos aspectos funcionais do sistema e de sua interface, estabeleceu-se percurso e tarefas a serem realizadas, partindo da página inicial do portal SENAI EAD até o acesso à primeira página do curso - o Ambiente Virtual de Aprendizagem. Durante a realização do percurso foram identificados e classificados questões relacionadas à usabilidade e o acesso às informações, acompanhado os critérios ergonômicos listados (Tabela 1), de acordo com a página específica, o conteúdo e a natureza dos problemas encontrados, como pode ser observado na Tabela 2. 
Vale explicar a identificação da natureza dos problemas encontrados. Identificam-se com o termo Programação, os problemas relacionados sobretudo à linguagem de programação, às instruções, ao sistema de bases de dados, às instruções e ao funcionamento do sistema operacional. Tais problemas tem relação com praticamente todos os critérios ergonômicos, mas é possível estabelecer articulação imediata com os critérios: Condução, Adaptabilidade, Gestão de Erros, Homogeneidade/Coerência e Compatibilidade. O termo conteúdo, identifica questões diretamente vinculadas à linguagem verbal - vocabulário, sintaxe e semântica textual. Tais questões mantém relação com todos os critérios ergonômicos, mas podemos destacar Condução, Carga de trabalho, Controle Explícito do Usuário, Homogeneidade/Coerência e, sobretudo Significado de Códigos. O termo Design, está relacionado aos problemas vinculados à configuração, ao uso e à organização de elementos gráfico-visuais, ao design da informação, o que implica, principalmente, na análise da organização e na hierarquia da informação. A natureza destes aspectos envolve todos os critérios ergonômicos apresentados na Tabela 1.

Tabela 2 - Problemas encontrados pelos avaliadores

\begin{tabular}{|c|c|c|c|}
\hline Problema & Página & Critério ergonômico violado & Natureza \\
\hline $\begin{array}{l}\text { Botão "anterior" não } \\
\text { funciona }\end{array}$ & 2 - Cadastro & Condução, gestão de erros & Design/Programação \\
\hline $\begin{array}{l}\text { Botões da barra lateral } \\
\text { não funcionam }\end{array}$ & 2 - Cadastro & Condução, gestão de erros & Design/Programação \\
\hline $\begin{array}{l}\text { Hierarquia do cadastro } \\
\text { confusa }\end{array}$ & $\begin{array}{l}\text { 3- Dados do } \\
\text { certificado }\end{array}$ & $\begin{array}{l}\text { Homogeneidade/coerência } \\
\text { gestão de erros }\end{array}$ & Design \\
\hline Página desnecessária & $\begin{array}{l}5 \text { - Dados do } \\
\text { responsável }\end{array}$ & Carga de trabalho & Design/Conteúdo \\
\hline $\begin{array}{l}\text { Sistema de comportou } \\
\text { de maneira inesperada }\end{array}$ & $6-$ Acesso ao AVA & $\begin{array}{l}\text { Homogeneidade/coerência, } \\
\text { gestão de erros }\end{array}$ & Design/Programação \\
\hline $\begin{array}{l}\text { Não compreendeu } \\
\text { hierarquia dos cursos }\end{array}$ & 7-Ambiente Virtual & $\begin{array}{l}\text { Homogeneidade/coerência, } \\
\text { condução, Significado de } \\
\text { códigos e denominações }\end{array}$ & Design/Conteúdo \\
\hline $\begin{array}{l}\text { Opção "busca" é muito } \\
\text { abrangente }\end{array}$ & 7-Ambiente Virtual & $\begin{array}{l}\text { Carga de Trabalho, } \\
\text { Significado de códigos e } \\
\text { denominações, } \\
\text { Homogeneidade/coerência }\end{array}$ & Conteúdo/Programação/Design \\
\hline $\begin{array}{l}\text { Falta de feedback ao se } \\
\text { inscrever no curso }\end{array}$ & 11 - Inscrição no curso & $\begin{array}{l}\text { Controle explícito do } \\
\text { usuário, Adaptabilidade, } \\
\text { Compatibilidade }\end{array}$ & Design/Programação \\
\hline Página desnecessária & 13 - Curso completo & Carga de trabalho & Design/Conteúdo \\
\hline
\end{tabular}

Fonte: autores.

No total, foram observados nove problemas pelos avaliadores e todos apresentam algum erro relacionado à interface e ao design das páginas. Para além da identificação dos erros apresentados durante o acesso, esta etapa de avaliação analítica permitiu estabelecer a lista de tarefas necessárias, para aplicação em processo de avaliação participativa com usuários do sistema. 


\subsection{Avaliação Participativa}

A metodologia proposta para a avaliação com os usuários, foi estabelecida a partir da combinação de ferramentas, que são habitualmente recomendadas em abordagens participativas. Essas ferramentas de avaliação são habitualmente aplicadas de maneira sistemática, com o intuito de melhorar a prática educativa (WANG; HANNAFIN, 2005 apud SANTA ROSA e STRUCHINER, 2010). Esta etapa privilegiou a obtenção de informações e dados sobre problemas encontrados pelos usuários participantes ao trabalhar com o sistema, para posterior comparação com os resultados obtidos na avaliação analítica. Embora este tipo de avaliação seja recomendado para as fases iniciais de projeto de interfaces, também pode ser usada em um sistema já existente, que deva ser melhorado ou ampliado (SANTA ROSA e MORAES, 2012).

Num primeiro momento foram selecionados os usuários, a partir de critérios embasados no perfil estabelecido pelo portal do SENAC EAD para o curso de Empreendedorismo. Adicionou-se a este perfil definido pela instituição, o interesse de estudantes de graduação de Design da região em fundamentos, conceitos, abordagens e ações relacionadas ao empreendedorismo e ligados, de alguma maneira, à indústria ou ao comércio do município onde vivem e trabalham. Deste modo, pôde-se reunir uma parcela de usuários estudantes do Curso de Design, com idade entre 19 e 21 anos, todos inseridos no cenário empresarial da cidade. Embora todos tivessem conhecimento sobre os cursos gratuitos oferecidos pelo SENAI, nenhum dos participantes já havia realizado algum dos cursos oferecidos pela instituição. Nesse sentido, os participantes não conheciam o sistema avaliado e puderam dar as suas primeiras impressões sobre a interface e o sistema.

Durante o processo de avaliação, buscou-se avaliar a qualidade da interação dos usuários com a interface, observando, sobretudo, a facilidade de uso, o que considerou a realização das tarefas estabelecidas e a compreensão e a satisfação dos usuários. Para tanto, o processo foi iniciado por avaliação cooperativa envolvendo usuários em ambiente controlado, a partir de teste de usabilidade. Nessas situações, as atividades e as tarefas realizadas pelos usuários são controladas, com o propósito de testar hipóteses, assim como observar ou medir comportamentos específicos durante a interação com a interface (MONK ET AL., 1993 apud SANTA ROSA e MORAES, 2012; PREECE ET AL., 2013). Assim, nesta etapa, todos os participantes utilizaram o mesmo equipamento, a mesma rede Wi-Fi em único laboratório.

A primeira parte da avaliação consistiu do uso do sistema, momento em que os usuários seguindo as tarefas estabelecidas buscaram realizar a matrícula e acessar a primeira página do AVA. Nesta fase, os usuários foram filmados individualmente realizando as tarefas propostas pelos avaliadores. Durante o processo, os usuários foram apontando as dificuldades encontradas e as dúvidas sobre o sistema. Após a etapa, os avaliadores conversaram individualmente com cada um dos usuários, buscando saber quais foram as suas impressões sobre o sistema.

A terceira fase da avaliação participativa consistiu em realização de grupo de foco, e compreendeu conversa com todos os usuários do sistema, oportunidade em que puderam em conjunto discutir as tarefas realizadas e debater sobre os entraves da interface.

Durante a conversa individual os estudantes comentaram sobre as suas dificuldades específicas, porém, quando discutiram sobre o sistema em grupo, puderam perceber outras questões, muitas delas enfrentadas por alguns dos participantes, mas que não foram registradas durante a execução das tarefas. 
Os resultados das fases da avaliação participativa, foram organizados, classificados, qualificados e quantificados. A tabela 3 apresenta os resultados.

Tabela 3 - Problemas encontrados pelos usuários

\begin{tabular}{|c|c|c|c|c|}
\hline Problema & Página & $\begin{array}{l}\text { Critério ergonômico } \\
\text { violado }\end{array}$ & Natureza & $\begin{array}{l}\text { Número de } \\
\text { usuários }\end{array}$ \\
\hline $\begin{array}{l}\text { Não encontrou o } \\
\text { botão "cadastrar- } \\
\text { se" }\end{array}$ & 1 - Site SENAI EAD & $\begin{array}{l}\text { Homogeneidade/coerência, } \\
\text { Significado de códigos e } \\
\text { denominações }\end{array}$ & Design/Conteúdo & 6 \\
\hline $\begin{array}{l}\text { Faltou informação } \\
\text { sobre formato de } \\
\text { senha }\end{array}$ & 2 - Dados de acesso & $\begin{array}{l}\text { Gestão de erros, } \\
\text { Significado de códigos e } \\
\text { denominações }\end{array}$ & Design/Conteúdo & 1 \\
\hline $\begin{array}{l}\text { Hierarquia do } \\
\text { cadastro confusa }\end{array}$ & $\begin{array}{l}\text { 3- Dados do } \\
\text { certificado }\end{array}$ & $\begin{array}{l}\text { Homogeneidade/coerência } \\
\text { gestão de erros }\end{array}$ & Design & 1 \\
\hline $\begin{array}{l}\text { O botão "anterior" } \\
\text { não funciona }\end{array}$ & $\begin{array}{l}3 \text { - Dados do } \\
\text { certificado } \\
5-\text { Termos de } \\
\text { compromisso }\end{array}$ & $\begin{array}{l}\text { Controle explícito do } \\
\text { usuário } \\
\text { Condução }\end{array}$ & Design/Programação & 6 \\
\hline $\begin{array}{l}\text { Campos de } \\
\text { preenchimento } \\
\text { ficam todos } \\
\text { vermelhos }\end{array}$ & $\begin{array}{l}4-\text { Dados } \\
\text { residenciais e } \\
\text { complementares }\end{array}$ & $\begin{array}{l}\text { Gestão de erros, } \\
\text { Homogeneidade/coerência, } \\
\text { Compatibilidade }\end{array}$ & Design/Programação & 3 \\
\hline $\begin{array}{l}\text { Muitas informações } \\
\text { na área de acesso }\end{array}$ & $\begin{array}{l}8-\text { Acesso ao } \\
\text { Ambiente Virtual }\end{array}$ & $\begin{array}{l}\text { Carga de trabalho } \\
\text { Adaptabilidade }\end{array}$ & Design/conteúdo & 1 \\
\hline $\begin{array}{l}\text { Não compreendeu } \\
\text { hierarquia dos } \\
\text { cursos }\end{array}$ & $\begin{array}{l}9-\text { Busca por } \\
\text { cursos gratuitos }\end{array}$ & $\begin{array}{l}\text { Homogeneidade/coerência, } \\
\text { condução, Significado de } \\
\text { códigos e denominações }\end{array}$ & Design/Conteúdo & 6 \\
\hline $\begin{array}{l}\text { Não compreendeu } \\
\text { os ícones } \\
\text { apresentados }\end{array}$ & $\begin{array}{l}10-\text { Busca por } \\
\text { cursos gratuitos } \\
11-\text { Inscrição no } \\
\text { curso }\end{array}$ & $\begin{array}{l}\text { Significado de códigos e } \\
\text { denominações }\end{array}$ & Design & 2 \\
\hline $\begin{array}{l}\text { Falta de feedback ao } \\
\text { se inscrever no } \\
\text { curso }\end{array}$ & $\begin{array}{l}11 \text { - Inscrição no } \\
\text { curso }\end{array}$ & $\begin{array}{l}\text { Condução, Controle } \\
\text { explícito do usuário, } \\
\text { Adaptabilidade, } \\
\text { Compatibilidade }\end{array}$ & Design/Programação & 1 \\
\hline $\begin{array}{l}\text { Não encontrou } \\
\text { opção "curso } \\
\text { completo", como } \\
\text { indicado no texto. }\end{array}$ & $\begin{array}{l}12-\text { Curso } \\
\text { completo }\end{array}$ & Condução & Design/Conteúdo & 2 \\
\hline $\begin{array}{l}\text { Texto de } \\
\text { apresentação não } \\
\text { atrativo }\end{array}$ & 12 - Início do curso & $\begin{array}{l}\text { Adaptabilidade, Significado } \\
\text { de códigos e denominações }\end{array}$ & Design & 2 \\
\hline $\begin{array}{l}\text { Interface com } \\
\text { muitos espaços } \\
\text { vazios e } \\
\text { informações difusas }\end{array}$ & 12 - Início do curso & $\begin{array}{l}\text { Homogeneidade/coerência } \\
\text { Carga de trabalho }\end{array}$ & Design & 1 \\
\hline $\begin{array}{l}\text { Página } \\
\text { desnecessária }\end{array}$ & $\begin{array}{l}12-\text { Curso } \\
\text { completo }\end{array}$ & Carga de trabalho & Design/Conteúdo & 6 \\
\hline
\end{tabular}

Fonte: autores. 
Nesta fase foram prospectados treze problemas durante o uso do sistema do SENAI EAD, todos relacionados a aspectos do design das páginas. Também é possível perceber que, oito dos problemas foram relatados por mais de um usuário, o que significa que não é apenas uma questão de uso individual do sistema, mas sim um ponto que pode ser corrigido na interface.

O estudo buscou comparar os resultados obtidos nos dois processos de avaliação - analítica e participativa, como segue.

\subsection{Comparativo entre as avaliações}

Ao final das duas etapas de avaliação, os avaliadores elaboraram uma quarta tabela, que contém uma lista com os dezesseis entraves relacionados ao sistema analisado. Nesta Tabela, podese identificar os problemas encontrados pelos próprios avaliadores, pelos usuários ou por ambos. Ainda, a Tabela 4 indica qual é a natureza desses problemas - Conteúdo, Design e Programação. É possível verificar, que alguns dos problemas levantados apresentam mais de uma natureza. Relacionar a natureza dos problemas foi importante para a pesquisa, pois foi possível, a partir desta análise, reconhecer as questões e aspectos diretamente relacionados com o design, possibilitando identificar oportunidades para a intervenção na interface ou no sistema, sugerindo possíveis soluções de projeto.

Tabela 4 - Problemas encontrados por avaliadores e usuários

\begin{tabular}{|c|c|c|c|}
\hline Problema & Avaliadores & Usuários & Natureza \\
\hline Não encontrou o botão "cadastrar-se" & & $\mathrm{X}$ & Design/Conteúdo \\
\hline $\begin{array}{l}\text { Faltou informação sobre formato de } \\
\text { senha }\end{array}$ & & $x$ & Design/Conteúdo \\
\hline Hierarquia do cadastro confusa & $\mathrm{x}$ & $\mathrm{X}$ & Design \\
\hline O botão "anterior" não funciona & $\mathrm{X}$ & $\mathrm{X}$ & Design/Programação \\
\hline $\begin{array}{l}\text { Campos de preenchimento ficam } \\
\text { todos vermelhos }\end{array}$ & & $x$ & Design/Programação \\
\hline Muitas informações na área de acesso & & & Design/Conteúdo \\
\hline $\begin{array}{l}\text { Não compreendeu hierarquia dos } \\
\text { cursos }\end{array}$ & $\mathrm{X}$ & $\mathrm{x}$ & Design/Conteúdo \\
\hline $\begin{array}{l}\text { Não compreendeu os ícones } \\
\text { apresentados }\end{array}$ & & $x$ & Design \\
\hline $\begin{array}{l}\text { Falta de feedback ao se inscrever no } \\
\text { curso }\end{array}$ & $x$ & $x$ & Design/Programação \\
\hline $\begin{array}{l}\text { Não encontrou opção "curso } \\
\text { completo", como indicado no texto. }\end{array}$ & & $x$ & Design/Conteúdo \\
\hline Texto de apresentação não atrativo & & $\mathrm{X}$ & Design \\
\hline $\begin{array}{l}\text { Interface com muitos espaços vazios e } \\
\text { informações difusas }\end{array}$ & & $\mathrm{X}$ & Design \\
\hline Página desnecessária & $\mathrm{X}$ & $\mathrm{X}$ & Design/Conteúdo \\
\hline Botões da barra lateral não funcionam & $x$ & & Design/Programação \\
\hline $\begin{array}{l}\text { Sistema de comportou de maneira } \\
\text { inesperada }\end{array}$ & $x$ & & Design/Programação \\
\hline Opção "busca" é muito abrangente & $\mathrm{x}$ & & Design/Conteúdo/Programação \\
\hline
\end{tabular}

Fonte: autores. 
Sobre os entraves, é possível perceber que as duas avaliações elucidaram uma grande quantidade de problemas nas páginas do cadastro até o primeiro acesso ao curso de Empreendedorismo do SENAI EAD, que afetam diretamente à usabilidade e ao acesso às informações nas páginas. $O$ fato das páginas oferecerem diversos problemas provocou, de acordo com o relato dos participantes, desinteresse e certa insegurança em realizar todo o processo de cadastro, inscrição e acesso ao AVA. Já na avaliação analítica tais problemas foram imediatamente relacionados a critérios mais técnicos e ergonômicos. Nesse caminho, foram levantados problemas relacionados especialmente à carga de trabalho, à condução e à gestão de erros.

A partir da avaliação com a participação dos usuários é possível afirmar que, embora o grupo de usuários fosse conciso, cada um possui suas particularidades ao interagirem com o sistema, revelando, assim, um número maior de dificuldades encontradas.

Desse modo, os avaliadores verificaram que, do total de dezesseis problemas, três deles foram levantados apenas pelos próprios avaliadores na avaliação analítica, enquanto sete, somente os usuários relataram. Os resultados dos dois processos de avaliação revelam, que tanto os avaliadores como os usuários observaram os seguintes problemas: botão 'anterior' não funcionou; hierarquia do menu com cursos disponíveis confusa; páginas desnecessárias durante o processo de cadastramento e matrícula no curso.

Embora parte das dificuldades tenham relação mais imediata com questões de programação e de conteúdo, todos podem ser resolvidos com soluções simples de design, baseadas principalmente em fundamentos da linguagem visual, como adequação de cores, uso de diagramas, organização e hierarquia de informações, diagramação. Entretanto, é válido lembrar que a interface do sistema do SENAI EAD funciona, apesar dos entraves e inconsistências encontradas durante a pesquisa. Igualmente, é importante mencionar que apenas soluções relacionadas ao design das interfaces não garantem o funcionamento adequado do sistema como um todo.

\section{Considerações finais}

O levantamento aqui apresentado revela um amplo universo para a pesquisa de design e para a aplicação de métodos de avaliação relacionadas ao uso articulado às funcionalidades das interfaces. Os estudos realizados sobre conceitos e fundamentos de Design Instrucional, design de interação, Design Centrado no Usuário [DCU] e usabilidade, atestaram a afirmação. Outrossim, a diversidade de métodos para avaliação de interfaces verificados no levantamento, confirmam a necessidade de analisar os processos, o que inclui a análise das fases e o uso de técnicas, ferramentas e instrumentos.

A proposta de comparar os métodos de avaliação aplicados neste estudo, com o propósito de estabelecer a eficiência das abordagens, analítica ou participativa, revelou que a articulação entre métodos e processos, flexibilizando as etapas e adaptando técnicas às necessidades específicas da pesquisa, devem ser consideradas, em especial nas abordagens centradas no usuário. Significa dizer, que um método não anula o outro, e, em muitos casos, os diferentes processos se complementam, possibilitando uma avaliação de interface mais abrangente e focada no usuário, enriquecendo o processo de investigação.

No contexto deste estudo, embora a avaliação participativa comparada ao método analítico tenha apresentado resultados mais abrangentes, que possibilitam maior oportunidade de 
redesenho do produto estudado, cada abordagem apresentou características distintas. O método analítico é, de fato, um processo mais técnico e de rápida aplicação. Já o processo participativo mantém maior relação com a realidade de uso dos usuários.

As avaliações de usabilidade nem sempre estão previstas no planejamento e desenvolvimento de projetos de sistemas e de interfaces, sobretudo no caso de equipes que não consideram os profissionais da área do design e especialistas em produção de conteúdo. De acordo com Prates e Barbosa (2003), muitas empresas ainda consideram um alto custo investir em testes de usabilidade, embora estudos comprovem que o retorno desse tipo de investimento é alto. Ignorar os testes de usabilidade é desconsiderar os fatores humanos relacionados aos produtos e serviços, e isso pode comprometer a adesão e o engajamento dos consumidores, dos usuários. Considerando o EAD e o Ambiente Virtual de Aprendizagem, esta questão é essencial.

\section{Referências}

CENSO EAD.br: relatório analítico de aprendizagem a distância no Brasil 2016. Curitiba: InterSaberes, 2017. Disponível em: http://abed.org.br/censoead2016/Censo_EAD_2016_portugues.pdf. Acesso em 20/11/2017.

FILATRO, A. Design Instrucional contextualizado: educação e tecnologia. São Paulo: Editora Senac, 2004.

Design Instrucional na prática. São Paulo: Pearson Education do Brasil, 2008.

GODOI, K. A.; PADOVANI, S. Avaliação de material didático digital centrada no usuário: uma investigação de instrumentos passíveis de utilização por professores. Produção, n. 3, p. 445-457, set/dez 2009.

INTERNATIONAL BOARD OF STANDARDS FOR TRAINING, PERFORMANCE AND INSTRUCTION. Instructional Design Competencies and Performance Statements. IBSTP org, 2012.

LOWDERMILK, T. Design centrado no usuário: um guia para desenvolvimento de aplicativos amigáveis. São Paulo: Novatec Editora, 2013.

MORÁN, J. Mudando a educação com metodologias ativas. In: SOUZA, C.A.; MORALES, O.E.T. (org.). Coleção Mídias Contemporâneas. Convergências Midiáticas, Educação e Cidadania: aproximações jovens. Vol. II. Ponta Grossa (PR): UEPG/PROEX, 2015, p. 15-33.

MUGNOL, M. A educação a distância no brasil: conceitos e fundamentos. In: Diálogo Educ., no 27, p.335-349, mai/ago 2009.

NIELSEN, JAKOB. Usability engineering. Boston: Academic Press,1993.

NIELSEN, J.; LORANGER, H. Usabilidade na Web: projetando websites com qualidade. Rio de Janeiro: Elservier, 2007.

PRATES, R.O.; BARBOSA, S.D.J. Avaliação de Interfaces de Usuário - Conceitos e Métodos. In: Anais do XXIII Congresso Nacional da Sociedade Brasileira de Computação. XXIII Jornadas de Atualização em Informática (JAI). SBC 2003, ago. 2003.

PREECE, J., ROGERS, Y.; SHARP, H.; PREECE, J. Design de interação: além da interação humanocomputador. Porto Alegre: Bookman, 2013.

SANTA ROSA, J.G.; STRUCHINER, M. Design Participativo de um Ambiente Virtual de Aprendizagem 
de Histologia. In: Revista Brasileira de Pesquisa em Educação em Ciências, no. 2, p. 1-19, mai/ago 2010.

SANTA ROSA, J. G.; MORAES, A. Avaliação e projeto no design de interfaces. Teresópolis: 2AB, 2012.

WHARTON, C.; RIEMAN, J.; LEWIS, C; POLSON, P. The Cognitive Walkthrough Method: a Practitioner's Guide. In: NIELSEN, J.; MACK, R.L. (eds.). Usability inspection methods. New York: John Wiley \& Sons, 1994. 